\title{
Stellar Distance and Velocity
}

\author{
Miloš Čojanović \\ Independent Scholar, Montreal, Canada \\ Email: cojmilmo@gmail.com
}

How to cite this paper: Čojanović, M. (2019) Stellar Distance and Velocity. Journal of Applied Mathematics and Physics, 7, 181-209.

https://doi.org/10.4236/jamp.2019.71016

Received: December 11, 2018

Accepted: January 22, 2019

Published: January 25, 2019

Copyright $\odot 2019$ by author(s) and Scientific Research Publishing Inc. This work is licensed under the Creative Commons Attribution International License (CC BY 4.0).

http://creativecommons.org/licenses/by/4.0/

\section{(c) (i) Open Access}

\begin{abstract}
In this paper, a method is presented by which it is possible to determine a distance between the sun and a star as well as a velocity at which the star moves relative to the sun. In order to achieve this, it is sufficient to know three positions of the star and the unit vectors determined by the star and three arbitrarily chosen points that do not lie on a single line. The method has been tested using the data generated by a computer program as well as real data obtained by Gaia mission. In the first case, we found the huge differences comparing the results derived by the method to the results calculated by the traditional parallax method. In the second case also, there are large differences between the obtained and the expected results, but primarily because of the form of the input data, that is not fully suited to the proposed method. Under certain conditions, one would be able to find a velocity at which the sun is moving regarding a stationary coordinate system $(K)$ that will be defined later on.
\end{abstract}

\section{Keywords}

Stellar Distance, Stellar Velocity

\section{Introduction}

Stellar parallax is defined as an apparent change in position of a star against the background of more distant objects, due to the movement of the earth revolving around the sun. In order to calculate a distance to the star, it is enough to know a distance from the earth to the sun that serves as a base line and the parallax angle that is obtained by two the measurements that have been made six months apart. There are some shortcomings of this method. Firstly, the base line is fixed thus the angles measured are always extremely small. Secondly, the base line is directly affected by the movement of the sun but it is not taken into the consideration. Thirdly, during the time of six months, a star is moving and changing its position which also affects a parallax angle. In some cases, a parallax has a negative value. It is believed that it may arise when the true parallax is smaller than 
its error. This assumption can only be partially correct, because some large negative parallaxes have been measured. In this novel method, sun and star movements regarding the stationary frame $(K)$ are taken into the calculation, which in certain cases could cause a negative parallax. The base line becomes a "base triangle" and its size is limited only by the time between the two measurements.

\section{Conversion Spherical Coordinates into Cartesian Coordinates}

First we will derive well known formulas for transformation from Spherical into Cartesian coordinate system and vice versa. Let suppose that a point $A$ is given by Spherical coordinates $(\lambda, \beta)$ (Figure 1 ).

$$
\begin{gathered}
\overline{S A}=1 \\
\lambda=\angle\left(S A_{x}, S A_{x y}\right) \\
\beta=\angle\left(S A_{x y}, S A\right) \\
a_{x}=\cos (\beta) * \cos (\lambda) \\
a_{y}=\cos (\beta) * \sin (\lambda) \\
a_{z}=\sin (\beta) \\
\overline{S A_{x}}=1 * a_{x} \\
\overline{S A_{y}}=1 * a_{y} \\
\overline{S A_{z}}=1 * a_{z} \\
\boldsymbol{a}=\left[a_{x}, a_{y}, a_{z}\right] \\
|\boldsymbol{a}|=1
\end{gathered}
$$

and vice versa if vector $\boldsymbol{a}=\left[a_{x}, a_{y}, a_{z}\right]$ is known then, we can easily find its Spherical coordinates.

$$
\begin{aligned}
& \beta=\arcsin \left(a_{z}\right) \\
& \lambda=\operatorname{atan} 2\left(\frac{a_{y}}{a_{x}}\right)
\end{aligned}
$$

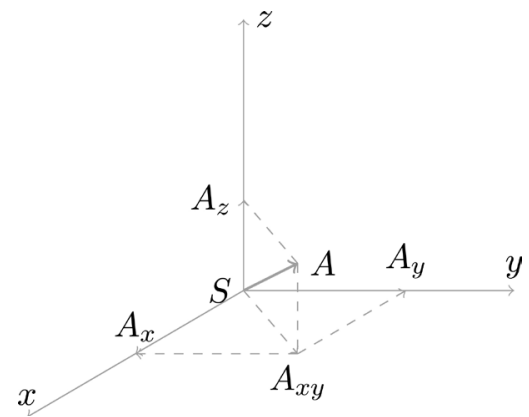

Figure 1. Transformation from spherical to cartesian coordinate system. 


\section{The Heliocentric-Ecliptic Coordinate System}

Let the $S(x, y, z)$ represents "The Heliocentric-Ecliptic Coordinate System" (Figure 2). Its origin $S$ is at the center of the sun and the fundamental plane $S(x, y)$ coincides with the "ecliptic", plane of the Earth's revolution about the sun. On the first day of Spring a line joining the center of the Earth and the center of the sun points in the direction of positive $x$-axis. This is called a vernal equinox direction.

The use of The Heliocentric-Ecliptic Coordinate System is obsolete, but in present paper we will use it for a better explanation of the proposed method.

Direction $S X_{0}$ represents Vernal equinox, and $t_{1}$ the time needed the Earth to move from the point $X_{0}$ to the point $A$. We can say that (Figure 2) depicts classical explanation how Earth revolves about the sun.

Now we will present a different interpretation of the same event. A stationary coordinate system marked as well by $(K)$ is joined to this referential frame. The coordinate system $(\mathrm{K})$ is not moving but rather remains fixed with respect to distant objects while the sun is moving by some velocity $v$ (Figure 3 ) regarding the coordinate system $(\mathrm{K})$.

In astronomy, an epoch is a moment in time used as a reference point, so we need to specify a certain time $T_{0}$ (TT-Terrestrial Time), which we are using as a reference. In other words an epoch is a moment for when a given position of an astronomical object is valid.

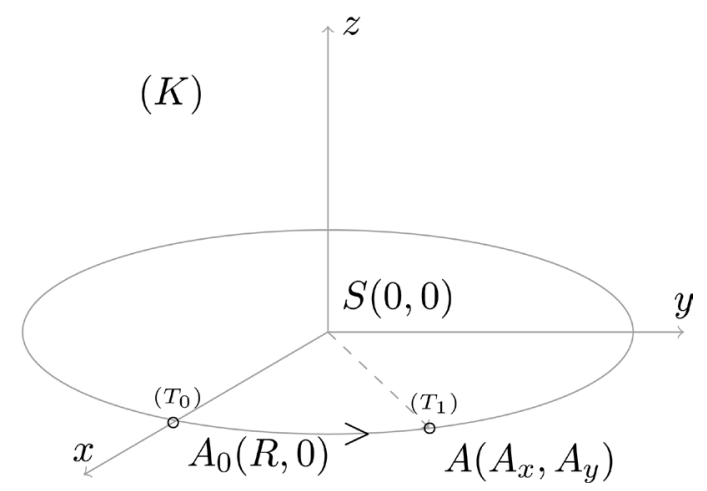

Figure 2. The position of the Earth in case the Sun is stationary regarding the (K).

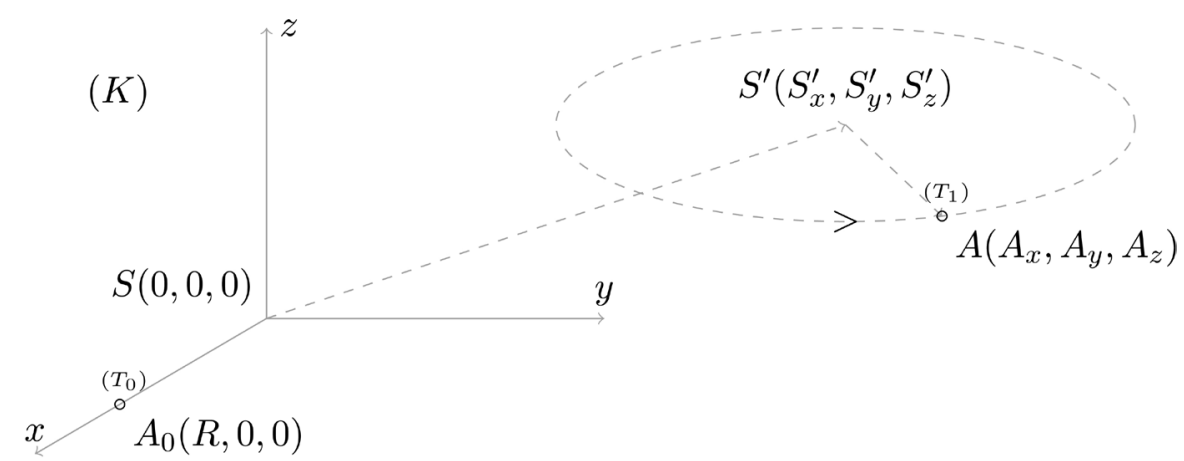

Figure 3. The position of the Earth in case the Sun is moving by velocity $v$ regarding the $(\mathrm{K})$. 
Finally we can suppose that for a certain period of time, a set of distant objects, the coordinate system $(K)$ and Epoch uniquely determine Stationary Reference Frame $(K)$. From Figure 2, it follows

$$
\begin{gathered}
A U=149597870.7 \mathrm{~km} \\
R=A U \\
\overline{S A_{0}}=R \\
\text { yearsec }=365.25 \times 24 \times 60 \times 60 \\
t_{1}=T_{1}-T_{0} \\
A_{x}=R * \cos \left(\frac{2 \Pi * t_{1}}{\text { yearsec }}\right) \\
A_{y}=R * \sin \left(\frac{2 \Pi * t_{1}}{\text { yearsec }}\right)
\end{gathered}
$$

From Figure 3, it follows

$$
\begin{gathered}
v=\left[v_{x}, v_{y}, v_{z}\right] \\
t_{1}=T_{1}-T_{0} \\
S_{x}^{\prime}=t_{1} * v_{x} \\
S_{y}^{\prime}=t_{1} * v_{y} \\
S_{z}^{\prime}=t_{1} * v_{z} \\
A_{x}=t_{1} * v_{x}+R * \cos \left(\frac{2 \Pi * t_{1}}{y e a r s e c}\right) \\
A_{y}=t_{1} * v_{y}+R * \sin \left(\frac{2 \Pi * t_{1}}{y e a r s e c}\right) \\
A_{z}=t_{1} * v_{z} \\
A_{x}=A_{x}\left(v_{x}, v_{y}, v_{z}\right) \\
A_{y}=A_{y}\left(v_{x}, v_{y}, v_{z}\right) \\
A_{z}=A_{z}\left(v_{x}, v_{y}, v_{z}\right)
\end{gathered}
$$

where $T_{1}$ (TT time) is expressed in seconds

Thus we have got the coordinates for the center of the Earth (marked by $A$ ) regarding the $(K)$. Assuming that the time $t_{1}$ is known from Equations (29)-(31) it follows that the coordinates of point $A$ can be expressed as a function of the velocity $\boldsymbol{v}$. For now $\boldsymbol{v}$ has been considered as a varible.

The astrometric processing uses a coordinate system known as the Barycentric Coordinate Reference System. It has its origin at the solar-system barycentre. Its axes are non-rotating with respect to objects at cosmological distances and coincide with those of the International Celestial Reference Frame.

The positions and proper motions of non-solar system objects derived from Gaia Data Release 1 observations are given in a reference frame that is aligned with the International Celestial Reference Frame (ICRF) [1]. ICRF coordinates are approximately the same as equatorial coordinates. 


\section{Coordinate Transformations}

The basis vectors in the equatorial system are here denoted $[x, y, z]$, The basis vectors in the ecliptic and galactic systems are respectively denoted $\left[x_{k}, y_{k}, z_{k}\right]$ and $\left[x_{g}, y_{g}, z_{g}\right]$. Thus, the arbitrary direction $u$ may be written in terms of the equatorial, ecliptic and galactic coordinates in the following way [2].

The transformation between the equatorial and ecliptic systems is given by:

$$
\left[x_{k}, y_{k}, z_{k}\right]=[x, y, z] * A K
$$

and

$$
[x, y, z]=\left[x_{k}, y_{k}, z_{k}\right] * A K^{\mathrm{T}}
$$

where

$$
\begin{aligned}
& \text { obliquity }=23.4392911 \text { (J2000.0 degrees) } \\
& \text { obliquity } 1=\frac{\text { obliquity } * \pi}{180}=0.409092802283074 \\
& A K=\left[\begin{array}{ccc}
1 & 0 & 0 \\
0 & \cos (\text { obliquity } 1) & -\sin (\text { obliquity } 1) \\
0 & \sin (\text { obliquity } 1) & \cos (\text { obliquity } 1)
\end{array}\right] \\
& =\left[\begin{array}{ccc}
1 & 0 & 0 \\
0 & 0.917482062146320 & -0.397777155753990 \\
0 & 0.397777155753990 & 0.9174820621463209
\end{array}\right] \\
& A K^{-1}=A K^{\mathrm{T}}=\left[\begin{array}{ccc}
1 & 0 & 0 \\
0 & 0.917482062146320 & 0.397777155753990 \\
0 & -0.397777155753990 & 0.9174820621463209
\end{array}\right]
\end{aligned}
$$

The transformation between the equatorial and galactic systems is given by:

$$
\left[x_{g}, y_{g}, z_{g}\right]=[x, y, z] * A G
$$

where:

$$
\begin{gathered}
A G=\left[\begin{array}{lll}
-0.0548755604 & +0.4941094279 & -0.8676661490 \\
-0.8734370902 & -0.4448296300 & -0.1980763734 \\
-0.4838350155 & +0.7469822445 & +0.4559837762
\end{array}\right] \\
A G^{-1}=A G^{\mathrm{T}}=\left[\begin{array}{llll}
-0.0548755604 & -0.8734370902 & -0.4838350155 \\
+0.4941094279 & -0.4448296300 & +0.7469822445 \\
-0.8676661490 & -0.1980763734 & +0.4559837762
\end{array}\right]
\end{gathered}
$$

The transformation between the galactic and ecliptic systems is given by:

$$
\begin{gathered}
{\left[x_{k}, y_{k}, z_{k}\right]=\left[x_{g}, y_{g}, z_{g}\right] * A G^{-1} * A K=\left[x_{g}, y_{g}, z_{g}\right] * A G^{\mathrm{T}} * A K} \\
{\left[x_{k}, y_{k}, z_{k}\right]=\left[x_{g}, y_{g}, z_{g}\right]} \\
*\left[\begin{array}{ccc}
-0.0548755604 & -0.9938213789915562 & -0.09647662628973946 \\
0.4941094279 & -0.1109907336202431 & 0.8622858750870472 \\
-0.867666149 & -3.515899626965191 E 10-4 & 0.4971471917263599
\end{array}\right](36)
\end{gathered}
$$


The peculiar motion of the Sun [3] with respect to the LSR is

$$
(U, V, W)=[11.1,12.24,7.25](\mathrm{km} / \mathrm{s})
$$

The velocity of the LSR about the center of the Milky Way is about 220 $(\mathrm{km} / \mathrm{sec})$, thus Sun velocity in Galactic coordinates is given by following equation

$$
\boldsymbol{v}_{g}=[11.1,232.24,7.25]
$$

Sun velocity in Ecliptic coordinates regarding the frame $(\mathrm{K})$

$$
\boldsymbol{v}_{k}=[107.85,-36.81,202.79]
$$

Of course, this can not be taken as a fully accurate value.

\section{Determining a Distance to a Star and Its Velocity regarding the Coordinate System (K)}

We assume that a star is moving with a uniform, rectilinear space motion $\boldsymbol{u}$ relative to the referential frame (K). At the some momemt $T$ (that will be derived later on) a signal has been emitted from the star (Figure 4). Postion of the star is marked by $A^{\prime}$. At the instant $t$ (Terrestrial Time) the signal has been recevied at the point $A$ on the Earth. Now we measure right ascension $(\alpha)$ and declination $(\delta)$ of the star regarding the equatorial coordinate system and transform them into the ecliptic longitude and ecliptic latitude $\left(\lambda_{1}, \beta_{1}\right)$ that can be also expressed as a unit vector $\boldsymbol{a}=\left[a_{x}, a_{y}, a_{y}\right]$ regarding the coordinate system $(K)$. In this way the direction in which the star lies has been determined. In order to find a position (in pollar coordinates) of the star at the moment $t$ we need to determine a distance between points $A$ and $A^{\prime}$.

Analogously we have the similiar situation with the pairs of points $\left(B, B^{\prime}\right)$ and $\left(C, C^{\prime}\right)$. Note that the points $A^{\prime}, B^{\prime}$ and $C^{\prime}$ are collinear while the points $A, B$ and $C$ form a triangle.

$$
\begin{aligned}
& d_{1}=\overline{A A^{\prime}} \\
& d_{2}=\overline{B B^{\prime}}
\end{aligned}
$$

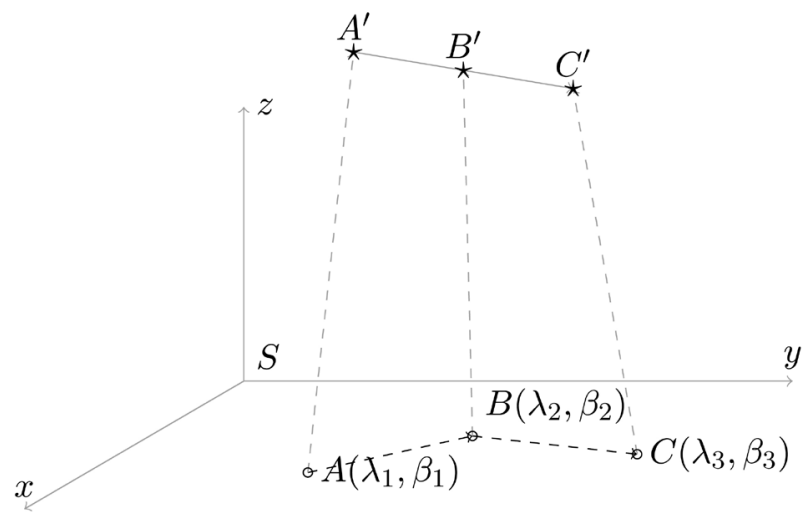

Figure 4. Three positions of a star and the corresponding Earth positions regarding the stationary coordinate system (K). 


$$
\begin{aligned}
& d_{3}=\overline{C C^{\prime}} \\
& \boldsymbol{a}=\left[a_{x}, a_{y}, a_{z}\right] \\
& a_{x}=\cos \left(\beta_{1}\right) * \cos \left(\lambda_{1}\right) \\
& a_{y}=\cos \left(\beta_{1}\right) * \sin \left(\lambda_{1}\right) \\
& a_{z}=\sin \left(\beta_{1}\right) \\
& \boldsymbol{b}=\left[b_{x}, b_{y}, b_{z}\right] \\
& b_{x}=\cos \left(\beta_{2}\right) * \cos \left(\lambda_{2}\right) \\
& b_{y}=\cos \left(\beta_{2}\right) * \sin \left(\lambda_{2}\right) \\
& b_{z}=\sin \left(\beta_{2}\right) \\
& \boldsymbol{c}=\left[c_{x}, c_{y}, c_{z}\right] \\
& c_{x}=\cos \left(\beta_{3}\right) * \cos \left(\lambda_{3}\right) \\
& c_{y}=\cos \left(\beta_{3}\right) * \sin \left(\lambda_{3}\right) \\
& c_{z}=\sin \left(\beta_{3}\right) \\
& A=\left(A_{x}, A_{y}, A_{z}\right) \\
& A_{x}=t_{1} * v_{x}+R * \cos \left(\frac{2 \Pi * t_{1}}{\text { yearsec }}\right) \\
& A_{y}=t_{1} * v_{y}+R * \sin \left(\frac{2 \Pi * t_{1}}{\text { yearsec }}\right) \\
& A_{z}=t_{1} * v_{z} \\
& B=\left(B_{x}, B_{y}, B_{z}\right) \\
& B_{x}=t_{2} * v_{x}+R * \cos \left(\frac{2 \Pi * t_{2}}{\text { yearsec }}\right) \\
& B_{y}=t_{2} * v_{y}+R * \sin \left(\frac{2 \Pi * t_{2}}{\text { yearsec }}\right) \\
& B_{z}=t_{2} * v_{z} \\
& C=\left(C_{x}, C_{y}, C_{z}\right) \\
& C_{x}=t_{3} * v_{x}+R * \cos \left(\frac{2 \Pi * t_{3}}{\text { yearsec }}\right) \\
& C_{y}=t_{3} * v_{y}+R * \sin \left(\frac{2 \Pi * t_{3}}{\text { yearsec }}\right) \\
& C_{z}=t_{3} * v_{z} \\
& A^{\prime}=\left(A_{x}^{\prime}, A_{y}^{\prime}, A_{z}^{\prime}\right) \\
& A_{x}^{\prime}=A_{x}+a_{x} * d_{1} \\
& A_{y}^{\prime}=A_{y}+a_{y} * d_{1}
\end{aligned}
$$




$$
\begin{aligned}
& A_{z}^{\prime}=A_{z}+a_{z} * d_{1} \\
& B^{\prime}=\left(B_{x}^{\prime}, B_{y}^{\prime}, B_{z}^{\prime}\right) \\
& B_{x}^{\prime}=B_{x}+b_{x} * d_{2} \\
& B_{y}^{\prime}=B_{y}+b_{y} * d_{2} \\
& B_{z}^{\prime}=B_{z}+b_{z} * d_{2} \\
& C^{\prime}=\left(C_{x}^{\prime}, C_{y}^{\prime}, C_{z}^{\prime}\right) \\
& C_{x}^{\prime}=C_{x}+c_{x} * d_{3} \\
& C_{y}^{\prime}=C_{y}+c_{y} * d_{3} \\
& C_{z}^{\prime}=C_{z}+c_{z} * d_{3}
\end{aligned}
$$

The points $A^{\prime}, B^{\prime}$ and $C^{\prime}$ are collinear therefore we can write a following expression.

$$
\boldsymbol{A}^{\prime} \boldsymbol{C}^{\prime}=k * \boldsymbol{A}^{\prime} \boldsymbol{B}^{\prime}
$$

where $k$ is for now an unknown coefficient.

$$
\begin{aligned}
& \boldsymbol{A}^{\prime} \boldsymbol{C}^{\prime}=\left[A_{x}^{\prime} C_{x}^{\prime}, A_{y}^{\prime} C_{y}^{\prime}, A_{z}^{\prime} C_{z}^{\prime}\right] \\
& \boldsymbol{A}^{\prime} \boldsymbol{B}^{\prime}=\left[A_{x}^{\prime} B_{x}^{\prime}, A_{y}^{\prime} B_{y}^{\prime}, A_{z}^{\prime} B_{z}^{\prime}\right] \\
& {\left[C_{x}+c_{x} * d_{3}-A_{x}-a_{x} * d_{1}, C_{y}+c_{y} * d_{3}-A_{y}-a_{y} * d_{1}, C_{z}+c_{z} * d_{3}-A_{z}-a_{z} * d_{1}\right]=(81)} \\
& k *\left[B_{x}+b_{x} * d_{2}-A_{x}-a_{x} * d_{1}, B_{y}+b_{y} * d_{2}-A_{y}-a_{y} * d_{1}, B_{z}+b_{z} * d_{2}-A_{z}-a_{z} * d_{1}\right] \\
& (k-1) * a_{x} * d_{1}-k * b_{x} * d_{2}+c_{x} * d_{3}=(1-k) * A_{x}+k * B_{x}-C_{x} \\
& (k-1) * a_{y} * d_{1}-k * b_{y} * d_{2}+c_{y} * d_{3}=(1-k) * A_{y}+k * B_{y}-C_{y} \\
& (k-1) * a_{z} * d_{1}-k * b_{z} * d_{2}+c_{z} * d_{3}=(1-k) * A_{z}+k * B_{z}-C_{z} \\
& {\left[\begin{array}{ccc}
(k-1) * a_{x} & -k * b_{x} & c_{x} \\
(k-1) * a_{y} & -k * b_{y} & c_{y} \\
(k-1) * a_{z} & -k * b_{z} & c_{z}
\end{array}\right]\left[\begin{array}{l}
d_{1} \\
d_{2} \\
d_{3}
\end{array}\right]=\left[\begin{array}{l}
(1-k) * A_{x}+k * B_{x}-C_{x} \\
(1-k) * A_{y}+k * B_{y}-C_{y} \\
(1-k) * A_{z}+k * B_{z}-C_{z}
\end{array}\right]} \\
& D=\left[\begin{array}{ccc}
(k-1) * a_{x} & -k * b_{x} & c_{x} \\
(k-1) * a_{y} & -k * b_{y} & c_{y} \\
(k-1) * a_{z} & -k * b_{z} & c_{z}
\end{array}\right] \\
& D_{0}=\left[\begin{array}{lll}
a_{x} & b_{x} & c_{x} \\
a_{y} & b_{y} & c_{y} \\
a_{z} & b_{z} & c_{z}
\end{array}\right] \\
& E=\left[\begin{array}{c}
(1-k) * A_{x}+k * B_{x}-C_{x} \\
(1-k) * A_{y}+k * B_{y}-C_{y} \\
(1-k) * A_{z}+k * B_{z}-C_{z}
\end{array}\right] \\
& \Delta=\operatorname{det}(D) \\
& \Delta_{0}=\operatorname{det}\left(D_{0}\right)
\end{aligned}
$$




$$
\begin{gathered}
\Delta=(-k) *(k-1) * \Delta_{0} \\
D_{1}=\left[\begin{array}{lll}
(1-k) * A_{x}+k * B_{x}-C_{x} & -k * b_{x} & c_{x} \\
(1-k) * A_{y}+k * B_{y}-C_{y} & -k * b_{y} & c_{y} \\
(1-k) * A_{z}+k * B_{z}-C_{z} & -k * b_{z} & c_{z}
\end{array}\right] \\
D_{2}=\left[\begin{array}{lll}
(k-1) * a_{x} & (1-k) * A_{x}+k * B_{x}-C_{x} & c_{x} \\
(k-1) * a_{y} & (1-k) * A_{y}+k * B_{y}-C_{y} & c_{y} \\
(k-1) * a_{z} & (1-k) * A_{z}+k * B_{z}-C_{z} & c_{z}
\end{array}\right] \\
D_{3}=\left[\begin{array}{lll}
(k-1) * a_{x} & -k * b_{x} & (1-k) * A_{x}+k * B_{x}-C_{x} \\
(k-1) * a_{y} & -k * b_{y} & (1-k) * A_{y}+k * B_{y}-C_{y} \\
(k-1) * a_{z} & -k * b_{z} & (1-k) * A_{z}+k * B_{z}-C_{z}
\end{array}\right]
\end{gathered}
$$

If $(\Delta \neq 0) \Leftrightarrow\left(\left(\Delta_{0} \neq 0\right)\right.$ and $(k \neq 0)$ and $\left.(k \neq 1)\right)$ then we have

$$
\begin{aligned}
& d_{1}\left(k, v_{x}, v_{y}, v_{z}\right)=\frac{\Delta_{1}}{\Delta}=\frac{\operatorname{det}\left(D_{1}\right)}{\operatorname{det}(D)} \\
& d_{2}\left(k, v_{x}, v_{y}, v_{z}\right)=\frac{\Delta_{2}}{\Delta}=\frac{\operatorname{det}\left(D_{2}\right)}{\operatorname{det}(D)} \\
& d_{3}\left(k, v_{x}, v_{y}, v_{z}\right)=\frac{\Delta_{3}}{\Delta}=\frac{\operatorname{det}\left(D_{3}\right)}{\operatorname{det}(D)}
\end{aligned}
$$

In order to calculate distances $d_{1}, d_{2}, d_{3}$ we have to determine a coefficient $k$.

Let $t_{1}$ denotes the time when signal is received at the point $A$. If $T_{1}$ denotes the time when the signal was being emitted from the star at the point $A^{\prime}$ we will have following equation

$$
T_{1}=t_{1}-\frac{d_{1}}{c}
$$

where $c$ denotes the speed of light in the reference frame $K$.

Analogously for the points $B$ and $B^{\prime}$ we will have

$$
T_{2}=t_{2}-\frac{d_{2}}{c}
$$

Now we have

$$
\begin{gathered}
\Delta T_{1}=T_{2}-T_{1}=t_{2}-t_{1}-\frac{d_{2}}{c}+\frac{d_{1}}{c} \\
\Delta T_{1}=\Delta t_{1}-\frac{d_{2}}{c}+\frac{d_{1}}{c} \\
\Delta T_{1}+\frac{d_{2}}{c}=\Delta t_{1}+\frac{d_{1}}{c}
\end{gathered}
$$

If $T_{3}$ denotes the time when the signal was being emitted from the star at the point $C^{\prime}$ and $t_{3}$ denoted the time when the signal is received at the point $C$ we will have following equation

$$
\Delta T_{2}=T_{3}-T_{1}=t_{3}-t_{1}-\frac{d_{3}}{c}+\frac{d_{1}}{c}
$$




$$
\begin{aligned}
& \Delta T_{2}=\Delta t_{2}-\frac{d_{3}}{c}+\frac{d_{1}}{c} \\
& \Delta T_{2}+\frac{d_{3}}{c}=\Delta t_{2}+\frac{d_{1}}{c}
\end{aligned}
$$

If $u$ denotes magnitude of the velocity $\boldsymbol{u}$ then from Equation (78) it follows

$$
\begin{gathered}
\overline{A^{\prime} B^{\prime}}=u * \Delta T_{1} \\
\overline{A^{\prime} C^{\prime}}=u * \Delta T_{2} \\
u * \Delta T_{2}=k *\left(u * \Delta T_{1}\right) \\
\Delta T_{2}=k * \Delta T_{1}
\end{gathered}
$$

Combining equations (102), (105) and (110) it follows that

$$
\begin{gathered}
\Delta t_{2}-\frac{d_{3}}{c}+\frac{d_{1}}{c}=k *\left(\Delta t_{1}-\frac{d_{2}}{c}+\frac{d_{1}}{c}\right) \\
d_{1} *(k-1)-d_{2} * k+d_{3}+\Delta t_{1} * c * k-\Delta t_{2} * c=0 \\
\frac{\Delta_{1}}{\Delta} *(k-1)-\frac{\Delta_{2}}{\Delta} * k+\frac{\Delta_{3}}{\Delta}+\Delta t_{1} * c * k-\Delta t_{2} * c=0 \\
\Delta_{1} *(k-1)-\Delta_{2} * k+\Delta_{3}-\Delta_{0} * \Delta t_{1} * c * k^{2} *(k-1)+\Delta_{0} * \Delta t_{2} * c * k *(k-1)=0
\end{gathered}
$$

Dividing Equation (114) by $k *(k-1)$ we obtain that

$$
\begin{aligned}
& \frac{\Delta_{1}}{k}-\frac{\Delta_{2}}{k-1}+\frac{\Delta_{3}}{k *(k-1)}-\Delta_{0} * \Delta t_{1} * c * k+\Delta_{0} * \Delta t_{2} * c=0 \\
& \Delta_{1}=\left|\begin{array}{lll}
(1-k) * A_{x}+k * B_{x}-C_{x} & -k * b_{x} & c_{x} \\
(1-k) * A_{y}+k * B_{y}-C_{y} & -k * b_{y} & c_{y} \\
(1-k) * A_{z}+k * B_{z}-C_{z} & -k * b_{z} & c_{z}
\end{array}\right|= \\
& \left|\begin{array}{ccc}
A_{x} & b_{x} & c_{x} \\
A_{y} & b_{y} & c_{y} \\
A_{z} & b_{z} & c_{z}
\end{array}\right| *(k-1) * k+\left|\begin{array}{ccc}
B_{x} & b_{x} & c_{x} \\
B_{y} & b_{y} & c_{y} \\
B_{z} & b_{z} & c_{z}
\end{array}\right| *\left(-k^{2}\right)+\left|\begin{array}{ccc}
C_{x} & b_{x} & c_{x} \\
C_{y} & b_{y} & c_{y} \\
C_{z} & b_{z} & c_{z}
\end{array}\right| * k \\
& \Delta_{1}=\Delta_{11} *(k-1) * k+\Delta_{12} *\left(-k^{2}\right)+\Delta_{13} * k \\
& \frac{\Delta_{1}}{k}=\Delta_{11} *(k-1)+\Delta_{12} *(-k)+\Delta_{13} \\
& \frac{\Delta_{1}}{k}=\left(\Delta_{11}-\Delta_{12}\right) * k+\left(\Delta_{13}-\Delta_{11}\right) \\
& \frac{\Delta_{1}}{k}=\left|\begin{array}{ccc}
A_{x}-B_{x} & b_{x} & c_{x} \\
A_{y}-B_{y} & b_{y} & c_{y} \\
A_{z}-B_{z} & b_{z} & c_{z}
\end{array}\right| * k+\left|\begin{array}{ccc}
C_{x}-A_{x} & b_{x} & c_{x} \\
C_{y}-A_{y} & b_{y} & c_{y} \\
C_{z}-A_{z} & b_{z} & c_{z}
\end{array}\right|=p_{1} * k+q_{1} \\
& \Delta_{2}=\left|\begin{array}{lll}
(k-1) * a_{x} & (1-k) * A_{x}+k * B_{x}-C_{x} & c_{x} \\
(k-1) * a_{y} & (1-k) * A_{y}+k * B_{y}-C_{y} & c_{y} \\
(k-1) * a_{z} & (1-k) * A_{z}+k * B_{z}-C_{z} & c_{z}
\end{array}\right|=
\end{aligned}
$$




$$
\begin{aligned}
& \left|\begin{array}{ccc}
a_{x} & A_{x} & c_{x} \\
a_{y} & A_{y} & c_{y} \\
a_{z} & A_{z} & c_{z}
\end{array}\right| *(k-1) *(1-k)+\left|\begin{array}{ccc}
a_{x} & B_{x} & c_{x} \\
a_{y} & B_{y} & c_{y} \\
a_{z} & B_{z} & c_{z}
\end{array}\right| * k(k-1)+\left|\begin{array}{ccc}
a_{x} & C_{x} & c_{x} \\
a_{y} & C_{y} & c_{y} \\
a_{z} & C_{z} & c_{z}
\end{array}\right| *(1-k)(12 \\
& \Delta_{2}=\Delta_{21} *(k-1) *(1-k)+\Delta_{22} * k *(k-1)+\Delta_{23} *(1-k) \\
& \frac{\Delta_{2}}{k-1}=\Delta_{21} *(1-k)+\Delta_{22} * k-\Delta_{23} \\
& \frac{\Delta_{2}}{k-1}=\left(\Delta_{22}-\Delta_{21}\right) * k+\left(\Delta_{21}-\Delta_{23}\right) \\
& \frac{\Delta_{2}}{k-1}=\left|\begin{array}{lll}
a_{x} & B_{x}-A_{x} & c_{x} \\
a_{y} & B_{y}-A_{y} & c_{y} \\
a_{z} & B_{z}-A_{z} & c_{z}
\end{array}\right| * k+\left|\begin{array}{ccc}
a_{x} & A_{x}-C_{x} & c_{x} \\
a_{y} & A_{y}-C_{y} & c_{y} \\
a_{z} & A_{z}-c_{z} & c_{z}
\end{array}\right|=p_{2} * k+q_{2} \\
& \Delta_{3}=\left|\begin{array}{lll}
(k-1) * a_{x} & -k * b_{x} & (1-k) * A_{x}+k * B_{x}-C_{x} \\
(k-1) * a_{y} & -k * b_{y} & (1-k) * A_{y}+k * B_{y}-C_{y} \\
(k-1) * a_{z} & -k * b_{z} & (1-k) * A_{z}+k * B_{z}-C_{z}
\end{array}\right|= \\
& \left|\begin{array}{ccc}
a_{x} & b_{x} & A_{x} \\
a_{y} & b_{y} & A_{y} \\
a_{z} & b_{z} & A_{z}
\end{array}\right| *(k-1)^{2} * k+\left|\begin{array}{ccc}
a_{x} & b_{x} & B_{x} \\
a_{y} & b_{y} & B_{y} \\
a_{z} & b_{z} & B_{z}
\end{array}\right| *(k-1) *(-k) * k+\left|\begin{array}{ccc}
a_{x} & b_{x} & C_{x} \\
a_{y} & b_{y} & C_{y} \\
a_{z} & b_{z} & C_{z}
\end{array}\right| *(k-1) * k \\
& \Delta_{3}=\Delta_{31} *(k-1)^{2} * k-\Delta_{32} *(k-1) * k^{2}+\Delta_{33} *(k-1) * k \\
& \frac{\Delta_{3}}{(k-1) * k}=\Delta_{31} *(k-1)-\Delta_{32} * k+\Delta_{33} \\
& \frac{\Delta_{3}}{(k-1) * k}=\left(\Delta_{31}-\Delta_{32}\right) * k+\left(\Delta_{33}-\Delta_{31}\right) \\
& \frac{\Delta_{3}}{(k-1) * k}=\left|\begin{array}{ccc}
a_{x} & b_{x} & A_{x}-B_{x} \\
a_{y} & b_{y} & A_{y}-B_{y} \\
a_{z} & b_{z} & A_{z}-B_{z}
\end{array}\right| * k+\left|\begin{array}{ccc}
a_{x} & b_{x} & C_{x}-A_{x} \\
a_{y} & b_{y} & C_{y}-A_{y} \\
a_{z} & b_{z} & C_{z}-A_{z}
\end{array}\right|=p_{3} * k+q_{3}
\end{aligned}
$$

And finally the Equation (115) can be written in the following form

$$
\begin{gathered}
A * k+B=0 \\
A=p_{1}-p_{2}+p_{3}-\Delta t_{1} * c * \Delta_{0} \\
B=q_{1}-q_{2}+q_{3}+\Delta t_{2} * c * \Delta_{0} \\
k\left(v_{x}, v_{y}, v_{z}\right)=-\frac{B\left(v_{x}, v_{y}, v_{z}\right)}{A\left(v_{x}, v_{y}, v_{z}\right)}
\end{gathered}
$$

If ( $A \neq 0$ ) then the Equation (134) has a unique solution, therefore the Equations (96)-(98) have unique solution, as well.

If $A, B$ and $C$ are collinear points, then it follows that

$$
\overrightarrow{A C}=\alpha * \overrightarrow{A B}
$$

It is easy to prove that $k=\alpha$, what implies that matrix $E=0$. In that case we would have only a trivial solution $d_{1}=d_{2}=d_{3}=0$.

The coefficient $k$ is eliminated from the equations, thus the distances 
$d_{1}, d_{2}, d_{3}$ are expressed only as a function of $\boldsymbol{v}$.

$$
\begin{aligned}
& d_{1}=d_{1}\left(v_{x}, v_{y}, v_{z}\right) \\
& d_{2}=d_{2}\left(v_{x}, v_{y}, v_{z}\right) \\
& d_{3}=d_{3}\left(v_{x}, v_{y}, v_{z}\right)
\end{aligned}
$$

We will see later how the change in the value of velocity $\boldsymbol{v}$ affects the distances $d_{1}, d_{2}, d_{3}$.

We assume that the coordinates of the points $A^{\prime}, B^{\prime}$ and $C^{\prime}$ and corresponding distances $d_{1}, d_{2}$ and $d_{3}$ are known thus we are able to determine a velocity $\boldsymbol{u}$ of the star regarding the frame (K). We have got the following equations.

$$
\begin{gathered}
\boldsymbol{u}=\left[u_{x}, u_{y}, u_{z}\right] \\
\boldsymbol{u}=\frac{\boldsymbol{A}^{\prime} \boldsymbol{C}^{\prime}}{\Delta T_{2}} \\
u_{x}\left(v_{x}, v_{y}, v_{z}\right)=\frac{C_{x}-A_{x}+c_{x} * d_{3}-a_{x} * d_{1}}{\Delta T_{2}} \\
u_{y}\left(v_{x}, v_{y}, v_{z}\right)=\frac{C_{y}-A_{y}+c_{y} * d_{3}-a_{y} * d_{1}}{\Delta T_{2}} \\
u_{z}\left(v_{x}, v_{y}, v_{z}\right)=\frac{C_{z}-A_{z}+c_{z} * d_{3}-a_{z} * d_{1}}{\Delta T_{2}} \\
|\boldsymbol{u}|=\sqrt{u_{x}^{2}+u_{y}^{2}+u_{z}^{2}}
\end{gathered}
$$

\section{The Case When $v=0$}

In this section it will be considered a case when $v$ is unknown. Unlike the unit vectors $\boldsymbol{a}, \boldsymbol{b}, \boldsymbol{c}$ that are obtained by measurements and remain unchanged, vector $\boldsymbol{v}$ will be substituted by 0 and $\boldsymbol{u}$ by $\Delta \boldsymbol{u}$. Therefore the formulas given in the [Section 5] get the new forms.

$$
\begin{gathered}
v_{x}=0 \\
v_{y}=0 \\
v_{z}=0 \\
\boldsymbol{A}=\left(A_{x}, A_{y}, A_{z}\right) \\
A_{x}=t_{1} * 0+R * \cos \left(\frac{2 \Pi * t_{1}}{\text { yearsec }}\right) \\
A_{y}=t_{1} * 0+R * \sin \left(\frac{2 \Pi * t_{1}}{\text { yearsec }}\right) \\
A_{z}=t_{1} * 0 \\
\boldsymbol{B}=\left(B_{x}, B_{y}, B_{z}\right)
\end{gathered}
$$




$$
\begin{gathered}
B_{x}=t_{2} * 0+R * \cos \left(\frac{2 \Pi * t_{2}}{\text { yearsec }}\right) \\
B_{y}=t_{2} * 0+R * \sin \left(\frac{2 \Pi * t_{2}}{\text { yearsec }}\right) \\
B_{z}=t_{2} * 0 \\
C=\left(C_{x}, C_{y}, C_{z}\right) \\
C_{x}=t_{3} * 0+R * \cos \left(\frac{2 \Pi * t_{3}}{\text { yearsec }}\right) \\
C_{y}=t_{3} * 0+R * \sin \left(\frac{2 \Pi * t_{3}}{\text { yearsec }}\right) \\
C_{z}=t_{3} * 0
\end{gathered}
$$

...Finally we have got

$$
\begin{aligned}
& d_{1} \approx d_{1}(0,0,0) \\
& d_{2} \approx d_{2}(0,0,0) \\
& d_{3} \approx d_{3}(0,0,0)
\end{aligned}
$$

...and

$$
\begin{aligned}
& \Delta u_{x} \approx \frac{C_{x}-A_{x}+c_{x} * d_{3}-a_{x} * d_{1}}{\Delta T_{2}} \\
& \Delta u_{y} \approx \frac{C_{y}-A_{y}+c_{y} * d_{3}-a_{y} * d_{1}}{\Delta T_{2}} \\
& \Delta u_{z} \approx \frac{C_{z}-A_{z}+c_{z} * d_{3}-a_{z} * d_{1}}{\Delta T_{2}}
\end{aligned}
$$

In that way we are able to determine approximate values for distances $d_{1}, d_{2}, d_{3}$ and star velocity $\Delta \boldsymbol{u}$ regarding the sun.

In the special case, instead of a star, we can observe the barycenter of the Galaxy. Then $\Delta \boldsymbol{u}$ denotes the velocity at which the barycenter of the Galaxy "moves" relative to the sun. In other words $\boldsymbol{v}_{g}=-\Delta \boldsymbol{u}$ denotes the velocity at which the Sun is moving about the barycenter of the Galaxy.

\section{Determining a Position of a Star}

In this section it will be explained how to find a position of the star at some instant $t_{2}$ if the following elements are known:

- $v$ velocity of the sun regarding the reference frame (K)

- $\quad \boldsymbol{u}$ velocity of a star regarding the reference frame $(\mathrm{K})$

- distance $d_{1}$ between the Earth and star at some instant $t_{1}$

- spherical coordinates of the star at point $A$ given by unit vector $\boldsymbol{a}=\left[a_{x}, a_{y}, a_{z}\right]$ at instant $t_{1}$

Thus our goal is to find the position of the star $B^{\prime}=\left(B_{x}^{\prime}, B_{y}^{\prime}, B_{z}^{\prime}\right)$ at instant $t_{2}$. From the (Figure 5) it follows that coordinates of the points $A^{\prime}$ and $B$ are 


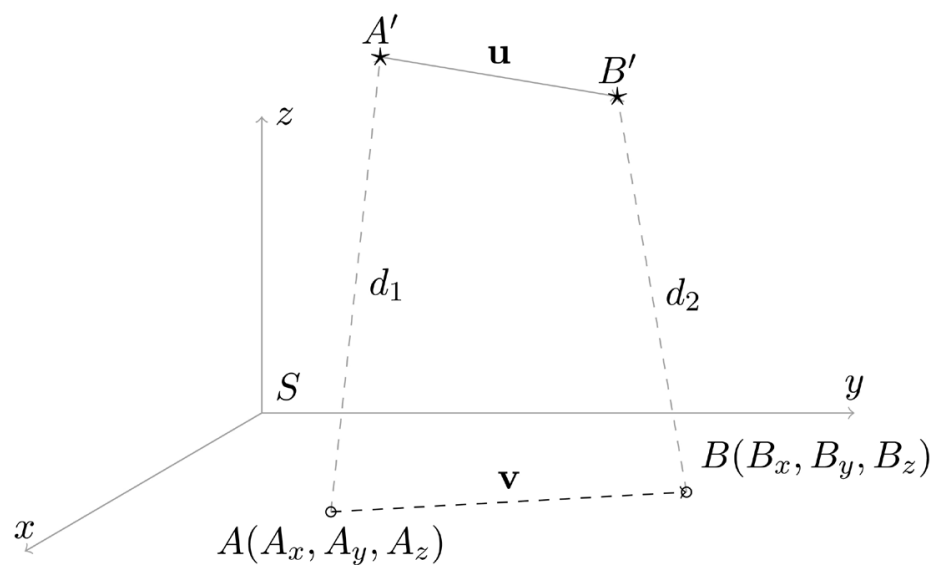

Figure 5. Two positions $A^{\prime}$ and $B^{\prime}$ of the star and the corresponding positions $A$ and $B$ of the Earth

known.

Therefore we get following equations

$$
\begin{aligned}
& \Delta t_{1}=t_{2}-t_{1} \\
& A^{\prime}=\left(A_{x}^{\prime}, A_{y}^{\prime}, A_{z}^{\prime}\right) \\
& A_{x}^{\prime}=A_{x}+a_{x} * d_{1} \\
& A_{y}^{\prime}=A_{y}+a_{y} * d_{1} \\
& A_{z}^{\prime}=A_{z}+a_{z} * d_{1} \\
& B=\left(B_{x}, B_{y}, B_{z}\right) \\
& B_{x}=A_{x}+\Delta t_{1} * v_{x} \\
& B_{y}=A_{y}+\Delta t_{1} * v_{y} \\
& B_{z}=A_{z}+\Delta t_{1} * v_{z} \\
& B^{\prime}=\left(B_{x}^{\prime}, B_{y}^{\prime}, B_{z}^{\prime}\right) \\
& B_{x}^{\prime}=A_{x}^{\prime}+\Delta T_{1} * u_{x} \\
& B_{y}^{\prime}=A_{y}^{\prime}+\Delta T_{1} * u_{y} \\
& B_{z}^{\prime}=A_{z}^{\prime}+\Delta T_{1} * u_{z} \\
& d_{2}^{2}=\Delta t_{1}^{2} * v^{2}+d_{1}^{2}+u^{2} * \Delta T_{1}^{2}-2 * \Delta t_{1} * d_{1} * \boldsymbol{v} * \boldsymbol{a} \\
&-2 * \Delta t_{1} * \boldsymbol{v} * \boldsymbol{u} * \Delta T_{1}+2 * d_{1} * \boldsymbol{a} * \boldsymbol{u} * \Delta T_{1}
\end{aligned}
$$

Obviously $\Delta T_{1}$ represents the time it takes for the star to move from the point $A^{\prime}$ to the point $B^{\prime}$. On the other hand we have

$$
\begin{gathered}
\frac{d_{1}}{c}+\Delta t_{1}=\frac{d_{2}}{c}+\Delta T_{1} \\
d_{2}=d_{1}+c * \Delta t_{1}-c * \Delta T_{1} \\
d_{2}^{2}=d_{1}^{2}+c^{2} * \Delta t_{1}^{2}+c^{2} * \Delta T_{1}^{2}+2 * d_{1} * c * \Delta t_{1} \\
-2 * d_{1} * c * \Delta T_{1}-2 * c^{2} * \Delta t_{1} * \Delta T_{1}
\end{gathered}
$$


From the Equations (183) and (186) we have

$$
\begin{aligned}
& \left(c^{2}-u^{2}\right) \Delta T_{1}^{2}-2\left(d_{1} c+c^{2} \Delta t_{1}-\Delta t_{1} \boldsymbol{u v}+d_{1} \boldsymbol{a} \boldsymbol{u}\right) \Delta T_{1} \\
& +\left(c^{2}-v^{2}\right) \Delta t_{1}^{2}+2 d_{1} \Delta t_{1}(c+\boldsymbol{v a})=0
\end{aligned}
$$

The Equation (187) can be written in the following short form and solved by an unknown $\Delta T_{1}$

$$
\begin{gathered}
A=c^{2}-u^{2} \\
B=d_{1} c+c^{2} \Delta t_{1}-\Delta t_{1} \boldsymbol{u} \boldsymbol{v}+d_{1} \boldsymbol{a} \boldsymbol{u} \\
C=\left(c^{2}-v^{2}\right) \Delta t_{1}^{2}+2 d_{1} \Delta t_{1}(c+\boldsymbol{v} \boldsymbol{a}) \\
A * x^{2}-2 * B * x+C=0 \\
x_{1,2}=\frac{B \pm \sqrt{B^{2}-A * C}}{A}
\end{gathered}
$$

There are two roots $x_{1}$ and $x_{2}$, but since $d_{2}>0$ it follows that

$$
\left(\frac{d_{2}}{c}=\frac{d_{1}}{c}+\Delta t_{1}-\Delta T_{1}>0\right) \Rightarrow\left(\frac{d_{1}}{c}+\Delta t_{1}>\Delta T_{1}\right)
$$

Thus we will choose a root of the Equation (191) for which this condition is fulfilled.

Now, when $\Delta T_{1}$ is known we are able to determine point $B^{\prime}$, distance $d_{2}$ and a unit vector $\boldsymbol{b}$.

$$
\begin{gathered}
d_{2}=B B^{\prime}=\sqrt{\left(B_{x}^{\prime}-B_{x}\right)^{2}+\left(B_{y}^{\prime}-B_{y}\right)^{2}+\left(B_{z}^{\prime}-B_{z}\right)^{2}} \\
b_{x}=\frac{B_{x}^{\prime}-B_{x}}{d_{2}} \\
b_{y}=\frac{B_{y}^{\prime}-B_{y}}{d_{2}} \\
b_{z}=\frac{B_{z}^{\prime}-B_{z}}{d_{2}} \\
\boldsymbol{b}=\left[b_{x}, b_{y}, b_{z}\right]
\end{gathered}
$$

Now we will transform the unit vector $\boldsymbol{a}=\left[a_{x}, a_{y}, a_{z}\right]$ from ecliptic to equatorial system. Transformed unit vector is marked by $\boldsymbol{a} \_\boldsymbol{e q}$.

$$
\begin{gathered}
\text { a_eq }=\left[a_{x}, a_{y}, a_{z}\right] * A K^{\mathrm{T}} \\
a_{x \_} e q=\boldsymbol{a} \_\boldsymbol{e q}[1,1] \\
a_{y \_} \text {eq }=\boldsymbol{a} \text { eq }[1,2] \\
a_{z} \text { eq }=\boldsymbol{a} \text { aeq }[1,3]
\end{gathered}
$$

By transformation from a right-angle coordinate system into the spherical coordinates we obtain the following equations

$$
\alpha_{1}=\operatorname{atan} 2\left(\frac{a_{y \_} e q}{a_{x \_} e q}\right)
$$




$$
\delta_{1}=\arcsin \left(a_{z-} e q\right)
$$

The same procedure will be repeated for unit vector $\boldsymbol{b}=\left[b_{x}, b_{y}, b_{z}\right]$.

$$
\begin{gathered}
\text { b_eq }=\left[b_{x}, b_{y}, b_{z}\right] * A K^{\mathrm{T}} \\
b_{x \_} e q=\boldsymbol{b} \_\boldsymbol{e q}[1,1] \\
b_{y \_} e q=\boldsymbol{b} \boldsymbol{e} \boldsymbol{e q}[1,2] \\
b_{z \_} e q=\boldsymbol{b} \_\boldsymbol{e q}[1,3] \\
\alpha_{2}=\operatorname{atan} 2\left(\frac{b_{y \_} e q}{b_{x \_} e q}\right) \\
\delta_{2}=\arcsin \left(b_{z \_} e q\right)
\end{gathered}
$$

where coordinates $\left(\alpha_{1}, \delta_{1}\right)$ and $\left(\alpha_{2}, \delta_{2}\right)$ represent positions of the star in the points $A$ and $B$ respectively.

We will define a proper motion $(\mu)$ as angular changes per year in the star's right ascension $\left(\mu_{R A}\right)$ and declination $\left(\mu_{D E C}\right)$, using a constant epoch. Now we will calculate proper motion components $\left(\mu_{R A}\right)$ and $\left(\mu_{D E C}\right)$.

Referring to (Figure 6) we get

$$
\begin{gathered}
S A^{\prime}=S D^{\prime}=S A=S D=1 \\
\alpha_{1}=\angle\left(x, S A^{\prime}\right) \\
\alpha_{2}=\angle\left(x, S D^{\prime}\right) \\
\Delta \alpha=\alpha_{2}-\alpha_{1}=\angle\left(A^{\prime} S D^{\prime}\right) \\
\delta_{1}=\angle\left(A^{\prime} S A\right)=\angle\left(D^{\prime} S D\right) \\
S^{\prime} A \| S A^{\prime} \\
S^{\prime} D \| S D^{\prime} \\
\mu_{R A}=\angle(S A, S D) \\
S^{\prime} A=S^{\prime} D=S A * \cos \left(\delta_{1}\right)=\cos \left(\delta_{1}\right) \\
\frac{A D}{2 * S^{\prime} A}=\sin \left(\frac{\Delta \alpha}{2}\right)
\end{gathered}
$$

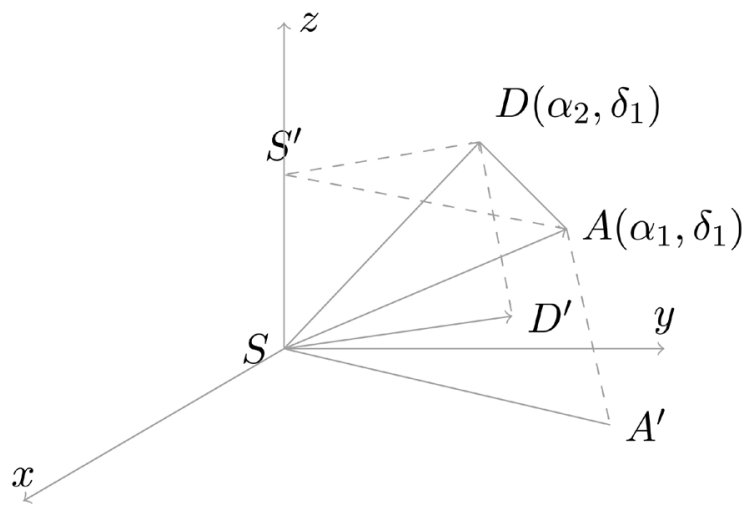

Figure 6. The component $\mu_{R A}$ of proper motion in the direction of right ascension. 


$$
\begin{gathered}
\frac{A D}{2}=S^{\prime} A * \sin \left(\frac{\Delta \alpha}{2}\right)=\cos \left(\delta_{1}\right) * \sin \left(\frac{\Delta \alpha}{2}\right) \\
\sin \left(\frac{\mu_{R A}}{2}\right)=\frac{A D}{2 * S A}=\frac{A D}{2}=\cos \left(\delta_{1}\right) * \sin \left(\frac{\Delta \alpha}{2}\right) \\
\mu_{R A}=2 * \arcsin \left(\cos \left(\delta_{1}\right) * \sin \left(\frac{\Delta \alpha}{2}\right)\right)
\end{gathered}
$$

Since $\Delta \alpha$ is a small angle it follows that

$$
\begin{gathered}
\arcsin \left(\cos \left(\delta_{1}\right) * \sin \left(\frac{\Delta \alpha}{2}\right)\right) \approx \cos \left(\delta_{1}\right) * \sin \left(\frac{\Delta \alpha}{2}\right) \approx \cos \left(\delta_{1}\right) *\left(\frac{\Delta \alpha}{2}\right) \\
\mu_{R A} \approx \cos \left(\delta_{1}\right) * \Delta \alpha=\cos \left(\delta_{1}\right) *\left(\alpha_{2}-\alpha_{1}\right)
\end{gathered}
$$

It is trivial to prove that

$$
\mu_{D E C}=\delta_{2}-\delta_{1}
$$

or if we expressed them in milliarcseconds we get

$$
\begin{aligned}
\mu_{R A}[\mathrm{mas}] & =\frac{\left(\mu_{R A}\right) \times(180 \times 3600 \times 1000)}{\Pi} \\
\mu_{D E C}[\mathrm{mas}] & =\frac{\left(\mu_{D E C}\right) \times(180 \times 3600 \times 1000)}{\Pi}
\end{aligned}
$$

\section{The Second Part}

In this section are given descriptions of four programs written in Maxima [4]. The purpose of their writing is testing the results obtained in the previous chapters. Instead of using the real measurements we will use data that are generated by a computer program. These data are presented in spherical coordinates $(\lambda, \beta)$ or as a corresponding unit vector $\boldsymbol{a}=\left[a_{x}, a_{y}, a_{z}\right]$ regarding the coordinate system $(\mathrm{K})$. We assume that velocities $\boldsymbol{v}$ and $\boldsymbol{u}$ as well a distance $d_{1}$ between the star and Earth at some instant $t$ are known.

Since the distances between the sun and stars are expressed by large numbers, 64-bits floating-point format which gives from 15 to 17 significant decimal digits is not sufficient to make enough precise arithmetic operations. Therefore, for correct and precise testing, a quad precision (128-bit or 34-digit) is required.

\section{Stellar parallax, proper motion and velocity}

If the vectors $\boldsymbol{b}=\left[b_{x}, b_{y}, b_{z}\right]$ and $\boldsymbol{c}=\left[c_{x}, c_{y}, c_{z}\right]$ are respectively corresponding unit vectors at points $B$ and $C$ [Figure 4] it is possible to calculate stellar parallax and its distance from the Earth using well known formulas. The time between two measurements is equal to six months.

$\mathrm{AU}$ is as usual defined as astronomical unit.

$$
\begin{gathered}
\text { parallax }=\frac{1}{2} * \arccos \left(\frac{\boldsymbol{b} \cdot \boldsymbol{c}}{\sqrt{\boldsymbol{b} \cdot \boldsymbol{b}} * \sqrt{\boldsymbol{c} \cdot \boldsymbol{c}}}\right) \\
\text { distance }=\frac{A U}{\sin (\text { parallax })}
\end{gathered}
$$


Since we work with approximate values, instead of $|\boldsymbol{b}|=1$ and $|\boldsymbol{c}|=1$ we will rather use $|\boldsymbol{b}|=\sqrt{\boldsymbol{b} \cdot \boldsymbol{b}}$ and $|\boldsymbol{c}|=\sqrt{\boldsymbol{c} \cdot \boldsymbol{c}}$

Let suppose that we observe a star from our Galaxy which has a following spherical coordinate regarding the equatorial coordinate system [label=]

- $\mathrm{RA}=12.56$

- $\mathrm{DEC}=-57.81$

These are field descriptions from the Table 1 and Table 2.

- $\boldsymbol{v}=\left[v_{x}, v_{y}, v_{z}\right]$-sun velocity $[\mathrm{km} / \mathrm{sec}]$

- $\boldsymbol{u}=\left[u_{x}, u_{y}, u_{z}\right]$-star velocity $[\mathrm{km} / \mathrm{sec}]$

- Real.dist - presumed distance between the star and Earth [Section 7] [km]

- PRX - parallax [Equation (228) [mas]]

- $\operatorname{Dist}(P R X)$-distance [Equation (229)] [km]

- Coeff - a ratio between the two distances, Coeff $=\frac{\operatorname{Dist}(P R X)}{\text { Realdist }}$

- PM.RA - proper motion (right ascension) [mas/year]

- PM.DEC - proper motion (declination) [mas/year]

By comparing the first and second rows, we can conclude that by changing only one component $u_{z}$ of the velocity $\boldsymbol{u}$ and keeping the values of all other elements unchanged, parallax and proper motion have been drastically changed. By comparing first, second and third rows, we can conclude that despite the same distances, but due to the different values for velocity $\boldsymbol{u}$, we obtain different values for the parallaxes. By comparing the first and fifth row, it follows that assuming that the sun is stationary, it does not significantly affect the value of the parallax nor the value of the proper motion.

It follows from the sixth row that when $\boldsymbol{u}=0$ and $\boldsymbol{v}=0$ it is possible directly calculated the distance by means of parallax.

Table 1. Parallax and proper motion of the star.

\begin{tabular}{ccccccccccccc}
\hline & $v_{x}$ & $v_{y}$ & $v_{z}$ & $u_{x}$ & $u_{y}$ & $u_{z}$ & Real.dist & PRX (mas) & Dist $($ PRX $)$ & Coeff & PM.RA & PM.DEC \\
\hline 1. & 107.852 & -36.81 & 202.79 & $v_{x}+40$ & $v_{y}+50$ & $v_{z}+60$ & $1.8 \mathrm{E} 15$ & 78.81 & $3.91 \mathrm{E} 14$ & 0.2175 & 214.70 & 233.63 \\
2. & 107.852 & -36.81 & 202.79 & $v_{x}+40$ & $v_{y}+50$ & $v_{z}-60$ & $1.8 \mathrm{E} 15$ & 7.6067 & $4.0565 \mathrm{E} 15$ & 2.2536 & 46.187 & -10.445 \\
3. & 107.852 & -36.81 & 202.79 & $v_{x}+80$ & $v_{y}+100$ & $v_{z}+120$ & $1.8 \mathrm{E} 15$ & 157.20 & $1.962 \mathrm{E} 14$ & 0.109 & 429.416 & 467.27 \\
4. & 107.852 & -36.81 & 202.79 & $v_{x}+80$ & $v_{y}+100$ & $v_{z}+120$ & $3.6 \mathrm{E} 15$ & 78.57 & $3.92719 \mathrm{E} 14$ & 0.109 & 214.7088 & 233.637 \\
5. & 0 & 0 & 0 & 40 & 50 & 60 & $1.8 \mathrm{E} 15$ & 78.78 & $3.91672 \mathrm{E} 14$ & 0.217595 & 214.7079 & 233.6343 \\
6. & 0 & 0 & 0 & 0 & 0 & 0 & $1.8 \mathrm{E} 14$ & 171.426 & $1.800001 \mathrm{E} 14$ & 1.00000055 & $-2.31 \mathrm{E}-9$ & $1.4 \mathrm{E}-8$ \\
\hline
\end{tabular}

Table 2. Proper motion of the star during the period of the six months.

\begin{tabular}{|c|c|c|c|c|c|c|c|c|c|c|c|}
\hline & $v_{x}$ & $v_{y}$ & $v_{z}$ & $u_{x}$ & $u_{y}$ & $u_{z}$ & $\operatorname{Dist}(A)$ & $\operatorname{Dist}(B)$ & $\operatorname{Dist}(C)$ & PM.RA. 5 & PM.DEC. 5 \\
\hline 1. & 107.852 & -36.81 & 202.79 & $v_{x}+40$ & $v_{y}+50$ & $v_{z}+60$ & $1.8 \mathrm{E} 15$ & 1.799999986 E15 & 1.799999972 E15 & 135.562 & 97.329 \\
\hline 2. & 107.852 & -36.81 & 202.79 & $v_{x}+40$ & $v_{y}+50$ & $v_{z}-60$ & $1.8 \mathrm{E} 15$ & $1.800001369 \mathrm{E} 15$ & 1.800002738 E15 & 51.30 & -24.71 \\
\hline
\end{tabular}


These are field descriptions from the Table 2.

- $\operatorname{Dist}(A)$-distance from the point $A$ to the star $[\mathrm{km}]$

- $\operatorname{Dist}(B)$-distance from the point $B$ to the star [km]

- $\operatorname{Dist}(C)$-distance from the point $C$ to the star $[\mathrm{km}]$

- PM.RA.5 - proper motion (right ascension) [mas $/\left(0.5^{\star}\right.$ year $\left.)\right]$

- DEC.RA.5 - proper motion (declination) [mas/(0.5*year)]

Comparing the first row of the table $T 1$ with the first row of the table $T 2$, we can conclude that the angular motion of the star in a period of one year is roughly twice as large as its angular motion in six months. Comparing the second row of the Table $T 1$ with the the second row of the Table $T 2$, we can conclude that the angular motion of a star over a period of six months is greater than the one in one year. So in this case it would appear that the star is moving zigzag.

Motion toward or away from the Sun called radial velocity is determined by using the Doppler Effect. Motion perpendicular to the direction to the Sun is called tangential velocity. It is accepted that transverse velocity $V_{T}$ is given by a following formula:

$$
V_{T}=k * \mu * d
$$

where distance is noted by $d$, proper motion is noted by $\mu$ and the factor $k$ comes from the unit conversion.

We are going to test the correctness of this formula.

Let the vector $\boldsymbol{v}$ represents the velocity of the sun and the vector $\boldsymbol{u}$ represents the velocity of the star regarding the (K). Relative velocity $\Delta \boldsymbol{u}=\left[\Delta u_{x}, \Delta u_{y}, \Delta u_{z}\right]$ of the star regarding the sun is given by following equations.

$$
\begin{gathered}
\Delta \boldsymbol{u}=\boldsymbol{u}-\boldsymbol{v} \\
\Delta u_{x}=u_{x}-v_{x} \\
\Delta u_{y}=u_{y}-v_{y} \\
\Delta u_{z}=u_{z}-v_{z}
\end{gathered}
$$

Let us, regarding the coordinate system $(\mathrm{K})$, define three unit vectors. The first vector noted by $\boldsymbol{z}$ _radial is directed to the star. Therefore, its spherical coordinates are $(\lambda, \beta)$, where $\lambda$ represents ecliptic longitude and $\beta$ represents ecliptic latitude. The second one marked by $\boldsymbol{x}_{\text {p }}$ m $\boldsymbol{m}$ long is determined by star proper motion in longitude direction. Its spherical coordinates are $(\lambda+\pi / 2,0)$. And the third one marked by $y_{\perp}$ pmlat will be determined by star proper motion in latitude direction. Its spherical coordinates are $(\lambda, \beta+\pi / 2)$.

$$
\begin{aligned}
\boldsymbol{x} \text { ppmlong }= & {[\cos (\lambda+\pi / 2) * \cos (0), \cos (0) * \sin (\lambda+\pi / 2), \sin (0)] } \\
y \text { pmlat }= & {[\cos (\lambda) * \cos (\beta+\pi / 2), \cos (\beta+\pi / 2) * \sin (\lambda), \sin (\beta+\pi / 2)] } \\
& \boldsymbol{z} \text { _radial }=[\cos (\lambda) * \cos (\beta), \cos (\beta) * \sin (\lambda), \sin (\beta)]
\end{aligned}
$$

In this way, a new coordinate system noted by $(K)$ has been defined. Its axes are determined by unit vectors $\left[\boldsymbol{x} \_\right.$pmlong, $y_{\perp}$ pmlat,$\left.z_{\text {_radial }}\right]$ and its origin is 
at the center of the sun. We can find the scalar projections of the vector $\Delta \boldsymbol{u}$

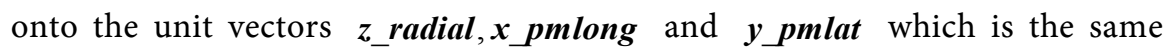
as to transform $\Delta \boldsymbol{u}$ from the coordinate system $(K)$ to the coordinate system $(K)$.

$$
\begin{gathered}
\Delta u \_l o n g=\boldsymbol{x} \_ \text {pmlong } * \Delta \boldsymbol{u} \\
\Delta u \_l a t=\boldsymbol{y} \_ \text {pmlat } * \Delta \boldsymbol{u} \\
\Delta u \_ \text {radial }=\boldsymbol{z} \_ \text {radial } * \Delta \boldsymbol{u}
\end{gathered}
$$

Let define $A Z$ a transformation matrix from coordinate system $(K)$ to the coordinate system $(K)$ by the following equation.

$$
\begin{aligned}
A Z & =\left[\begin{array}{ccc}
\cos (\lambda+\pi / 2) * \cos (0) & \cos (0) * \sin (\lambda+\pi / 2) & \sin (0) \\
\cos (\lambda) * \cos (\beta+\pi / 2) & \cos (\beta+\pi / 2) * \sin (\lambda) & \sin (\beta+\pi / 2) \\
\cos (\lambda) * \cos (\beta) & \cos (\beta) * \sin (\lambda) & \sin (\beta)
\end{array}\right] \\
& =\left[\begin{array}{ccc}
-\sin (\lambda) & \cos (\lambda) & 0 \\
-\cos (\lambda) * \sin (\beta) & -\sin (\beta) * \sin (\lambda) & \cos (\beta) \\
\cos (\lambda) * \cos (\beta) & \cos (\beta) * \sin (\lambda) & \sin (\beta)
\end{array}\right]
\end{aligned}
$$

By transforming the velocity $\Delta \boldsymbol{u}$ from the coordinate system $(K)$ to the coordinate system $(K)$, we obtain the following equation

$$
\begin{gathered}
{\left[\Delta u \_l o n g, \Delta u \_l a t, \Delta u \_ \text {radial }\right]=\Delta \boldsymbol{u} * A Z^{\mathrm{T}}} \\
\Delta u \_ \text {pmlong }=\frac{d * \tan (P M \cdot L O N G)}{\Delta T_{2}} \\
\Delta u \_ \text {pmlat }=\frac{d * \tan (P M \cdot L A T)}{\Delta T_{2}}
\end{gathered}
$$

where distance between the sun and a star is noted by $d$ and $\Delta T_{2}$ is defined by Equation (105).

If the formula given by the Equation (230) is valid then we should have that

$$
\begin{gathered}
\Delta u \_l o n g=\Delta u \_p m l o n g \\
\Delta u \_l a t=\Delta u \_p m l a t
\end{gathered}
$$

From the two examples shown in Table 3, we can see that the conditions given by (246) and (247) are met.

Matrix $A Z$ is an orthogonal matrix. As a linear transformation, an orthogonal matrix preserves the dot product of vectors. In other words orthogonal transformations preserve lengths of vectors and angles between them. Let PM.LONG

Table 3. Transverse velocity of a star determined in two ways.

\begin{tabular}{cccccccccccc}
\hline & $\Delta u_{x}$ & $\Delta u_{y}$ & $\Delta u_{z}$ & $\Delta u_{-}$long & $\Delta u_{-}$lat & $\Delta u_{-}$radial & Distance & PM.LONG & PM.LAT & $\Delta u \_p m l o n g$ & $\Delta u \_p m l a t$ \\
\hline 1. & 10 & 20 & 30 & 8.740 & 35.5236 & -7.8528 & $1.8 \mathrm{E} 15$ & 31.6001 & 128.4824 & 8.73817 & 35.52842 \\
2. & -30 & 20 & -10 & 34.67859 & -14.0384 & 0.5627 & $1.8 \mathrm{E} 15$ & 125.406 & -50.767 & 34.6788 & -14.03868 \\
\hline
\end{tabular}


and PM.LAT denote components for proper motion of the star in the ecliptic coordinate system $(\mathrm{K})$. Then we have the following equation.

$$
\mu=\sqrt{P M \cdot R A^{2}+P M \cdot D E C^{2}}=\sqrt{P M \cdot L O N G^{2}+P M \cdot L A T^{2}}
$$

The differences between fields $\Delta u$ pmlong and $\Delta u$ long and fields $\Delta u \_p m l a t$ and $\Delta u$ lat are minimal, and therefore we can say that the formula given by (230) is correct. Of course, this is not a rigorous proof, but a conclusion that resulted from a few examples.

At the end of this section let us suppose that $\Delta u$ pmlong, $\Delta u$ p pmlat and radial velocity noted by $\Delta u$ radial are known. Transforming the velocity [ $\Delta u \_$pmlong, $\Delta u \_$pmlat, $\Delta u \_$radial $]$from the coordinate system $(K)$ to the coordinate system $(K)$ we get the velocity $\Delta \boldsymbol{u}^{\prime}$ defined by following equation.

$$
\Delta \boldsymbol{u}^{\prime}=\left[u_{x}^{\prime}, u_{y}^{\prime}, u_{z}^{\prime}\right]=\left[\Delta u \_ \text {pmlong }, \Delta u \_ \text {pmlat }, \Delta u_{\_} \text {radial }\right] * A Z
$$

In this way we have calculated the velocity of the star regarding the sun in two different ways.

The first method is given by the Equations (166)-(168), and the second one by Equation (249)

$$
\begin{gathered}
\Delta \boldsymbol{u}=\Delta \boldsymbol{u}(\boldsymbol{a}, \boldsymbol{b}, \boldsymbol{c}) \\
\Delta \boldsymbol{u}^{\prime}=\Delta \boldsymbol{u}^{\prime}(d, \mu, \text { radial_velocity })
\end{gathered}
$$

If $\Delta \boldsymbol{u}^{\prime} \approx \Delta \boldsymbol{u}$ then we can conclude that measurements and proposed methodology are correct. Please refer to the attached program "parallax0.wxmx" for further details, investigation and testing.

Distance and relative velocity of a star if we assume that the sun is stationary regarding the frame $(\mathrm{K})$

In this case we assume that $\boldsymbol{v}=0$ and star velocity $\boldsymbol{u}$ is substituted by its relative velocity $\Delta \boldsymbol{u}$ regarding the sun.

Let suppose that we observe a star which has a following spherical coordinate regarding the ecliptic coordinate system $(\mathrm{K})$ [label=]

- $\quad \mathrm{LONG}=29.45$

- $\mathrm{LAT}=60.58$

Star relative velocity $\Delta \boldsymbol{u}$ is fixed and defined by the following equations

$$
\begin{aligned}
\Delta u_{x} & =-10 \\
\Delta u_{y} & =-30 \\
\Delta u_{z} & =30
\end{aligned}
$$

These are fields descriptions from the (Table 4).

- $t_{1}$-time[years] when the first measurement at point $A$ was performed

- $t_{2}$-time[years] when the second measurement at point $B$ was performed

- $t_{3}$-time [years] when the third measurement at point $C$ was performed

- Realdist - presumed distance [km] between the star and Earth [Section 7]

- Calc.dist - distance (km) Equation (177)

- Diff $=$ Calc.dist - Realdist 
Table 4. The distances in the case that the sun is stationary.

\begin{tabular}{cccccccccc}
\hline & $t_{1}$ & $t_{2}$ & $t_{3}$ & Real.dist & Calc.dist & Diff & $d u_{x}$ & $d u_{y}$ & $d u_{z}$ \\
\hline 1. & 0 & $4 / 12$ & $8 / 12$ & $4.7 \mathrm{E} 14$ & $4.699999994915726 \mathrm{E} 14$ & $-5.08427 \mathrm{E} 05$ & -10.02 & -30.00 & 29.95 \\
2. & 0 & $28 / 12$ & $56 / 12$ & $4.7 \mathrm{E} 14$ & $4.699999575402733 \mathrm{E} 14$ & $-4.24597 \mathrm{E} 07$ & -9.99 & -29.99 & 30.01 \\
3. & 0 & $40 / 12$ & $80 / 12$ & $4.7 \mathrm{E} 14$ & $4.699999142379139 \mathrm{E} 14$ & $-8.5762 \mathrm{E} 07$ & -9.99 & -29.99 & 30.01 \\
4. & 0 & $76 / 12$ & $152 / 12$ & $4.7 \mathrm{E} 14$ & $4.699996938037172 \mathrm{E} 14$ & $-3.06196 \mathrm{E} 08$ & -9.99 & -29.99 & 30.01 \\
\hline
\end{tabular}

- $d u_{x}$-component $\Delta u_{x}$ calculated by computer program

- $d u_{y}$-component $\Delta u_{y}$ calculated by computer program

- $d u_{z}$-component $\Delta u_{z}$ calculated by computer program

We can conclude from the obtained results that the increase in the time between the two measurements negatively affects the accuracy in determining the distance, while the accuracy in determining relative velocity remains relatively constant. In addition, we can make assumption that this method is much more precise in determining the distance compared to the "traditional" parallax method.

Please refer to the attached program "distance_velocity_0.wxmx" for further investigation and testing.

Determining distance and velocity of a star in case that velocity $v$ of the sun regarding the frame $(\mathrm{K})$ is known

Again let us suppose that $(\mathrm{K})$ is stationary coordinate system regarding the galaxy barycenter and that the sun moves at velocity $v=\left[v_{x}, v_{y}, v_{z}\right]$ regarding the $(\mathrm{K})$. Velocity of the sun is given by Equation (38).

- $v_{x}=107.852[\mathrm{~km} / \mathrm{sec}]$

- $v_{y}=-36.81[\mathrm{~km} / \mathrm{sec}]$

- $v_{z}=202.79[\mathrm{~km} / \mathrm{sec}]$

We observe a star which has a following spherical coordinate regarding the ecliptic coordinate system $(\mathrm{K})$

- $\mathrm{LONG}=29.45$

- $\mathrm{LAT}=60.58$

Star velocity $\boldsymbol{u}$ regarding the $(\mathrm{K})$ is given by the following equations.

$$
\begin{gathered}
u_{x}=97.85 \\
u_{y}=-66.81 \\
u_{z}=232.79
\end{gathered}
$$

These are field descriptions from the Table 5.

- $t_{1}$-time[year] when the first measurement at point $A$ was made

- $t_{2}$-time[year] when the second measurement at point $B$ was made

- $t_{3}$-time [year] when the third measurement at point $C$ was made

- Realdist - presumed distance [ $\mathrm{km}$ ) between the star and Earth [Section 7]

- Calc.dist - distance [km] Equation (130)

- Diff $=$ Calc.dist - Realdist 
Table 5. The distances between the star and Earth when velocity $\boldsymbol{v}$ of the sun regarding the $(\mathrm{K})$ is known.

\begin{tabular}{|c|c|c|c|c|c|c|c|c|c|}
\hline & $t_{1}$ & $t_{2}$ & $t_{3}$ & Real.dist & Calc.dist & Diff & $u_{x}^{\prime}$ & $u_{y}^{\prime}$ & $u_{z}^{\prime}$ \\
\hline 1. & 0 & $4 / 12$ & $8 / 12$ & 4.7 E14 & 4.70000002962370 E14 & 2.96237 E06 & 97.85 & -66.81 & 232.79 \\
\hline 2. & 0 & $28 / 12$ & $56 / 12$ & 4.7 E14 & 4.69999999883636 E14 & -1.16363 E05 & 97.85 & -66.81 & 232.79 \\
\hline 3. & 0 & $40 / 12$ & $80 / 12$ & 4.7 E14 & 4.70000000121683 E14 & $1.21683 \mathrm{E} 05$ & 97.85 & -66.81 & 232.79 \\
\hline
\end{tabular}

- $u_{x}^{\prime}$-component $u_{x}$ calculated by the program

- $u_{y}^{\prime}$-component $u_{y}$ calculated by the program

- $u_{z}^{\prime}$-component $u_{z}$ calculated by the program

Comparing the first row of the Table 5 with the second and third one and Comparing the second and the third row with the fourth row of the Table 5 we can conclude that increasing the interval between two measurements increases the accuracy in determining the distance $d$. This is completely contrary to the situation in the previous section. We can say that the errors in this case are negligible and that they are due to rounding up the numbers into 64-bit format.

Please refer to the attached program "distance_velocity_v.wxmx" for further explanations and data testing.

\section{Discussion}

Based on what we have found so far, we can conclude that assuming that $\boldsymbol{v}=0$, the obtained results are far more reliable than the data obtained by the "parallax" method. Problems arise when we increase the interval between two measurements. Increasing this interval increases the error in distance calculation.

Method in which the velocity $\boldsymbol{v}$ is known gives the best results. In addition, by increasing the interval between the two measurements, the angles between the unit vectors $\boldsymbol{a}, \boldsymbol{b}$ and $\boldsymbol{c}$ are increasing which improves the accuracy of the calculations.

Only in cases when the velocity $\boldsymbol{v}$ is known and the measurements have been carried out over a sufficiently long period of time we can obtain reliable data on the distance $d$ and velocity $\boldsymbol{u}$.

These conclusions should be taken with a certain reservation because they are based only on a few examples.

\section{Testing the Proposed Method Using Data Obtained from Gaia Catalogs DR1 and DR2}

The reference system for the source catalogs is the barycentric celestial reference system (BCRS/ICRS). Observations are more naturally expressed in the centre-of-masses reference system (CoMRS) which is defined from the BCRS by special relativistic coordinate transformations. This system moves with the Gaia spacecraft and is defined to be kinematically non-rotating with respect to the BCRS/ICRS [5]. 
In order to successfully apply the described method, we have to know the unit vectors and the positions of the detector. The exact location of the satellite will be substituted by the center of the earth and the unit vectors will be derived from the data given in the Gaia's catalogs [6] (option "Search"). We should also keep in mind that we need spherical coordinates of a star regarding the coordinate system with its origin at the satellite instead of the sun barycenter.

From the Gaia catalogs we can directly get the following data

- RA2015-Barycentric right ascension of the source in ICRS at the reference epoch J2015.0

- DEC2015-Barycentric declination of the source in ICRS at the reference epoch $\mathrm{J} 2015.0$

- $R A 2015: 5-$ Barycentric right ascension of the source in ICRS at the reference epoch $\mathrm{J} 2015.5$

- DEC2015:5-Barycentric declination of the source in ICRS at the reference epoch J2015.5

- PRX.2015:5-Absolute stellar parallax of the source at the reference epoch $\mathrm{J} 2015.5$

- PM.RA-Proper motion in right ascension direction, J2015.5 epoch

- PM.DEC-Proper motion (Declination) J2015.5 epoch

From the obtained data we can derive a right ascension $R A 2016.5$ and a declination $D E C 2016.5$ of a star at the reference epoch J2016.5.

$$
\begin{gathered}
R A 2016.5=R A 2015.5+P M . R A * \cos (D E C 2015.5) \\
D E C 2016.5=D E C 2015.5+P M . D E C
\end{gathered}
$$

Vernal equinox in 2015 happened on March Mar 20, 22:45 (GMT) or 78.91666667 days from the beginning of the year 2015.

The reference epoch for Gaia DR1 is J2015

J2015 = 2015 Jan 1, 00:00:00 (?)

The reference epoch for Gaia DR2 is J2015.5

J2015.5 = 2015 July 2, 21:00:00

$t_{0}=-(78.91666667 /$ year $) *$ year_sec the time of the first measurement (an epoch J2015.0)

$t_{1}=-(0.5-78.91666667 /$ year $) *$ year_sec the time of the second measurement (an epoch J2015.5)

$t_{2}=-(1.5-78.91666667 /$ year $) *$ year_sec the time of the third measurement (an epoch J2016.5)

We will assume that the measurement were taken regarding the Equatorial coordinate system with its origin at the center of the Earth.

In this way, we obtained all the parameters needed to calculate a star relative velocity regarding the sun and the star distance in two different ways.

- Distance $(P R X)$-Distance to a star calculated by using traditional parallax method

- Distance $(v)$-Distance to a star, assuming that $v=0$

- $u_{x}^{\prime}$-determined by Equation (249) 
- $\quad u_{y}^{\prime}$-determined by Equation (249)

- $u_{z}^{\prime}$-determined by Equation (249)

- $u_{x}$-determined by Equation (166)

- $u_{y}$-determined by Equation (167)

- $u_{z}$-determined by Equation (168)

The stars are chosen at random. The results are summarized in the two following tables.

The correctness of the proposed method and the accuracy of the input parameters can be tested in the following way.

Firstly, we can compare the $\Delta \boldsymbol{u}=\left[u_{x}, u_{y}, u_{z}\right]$ Equation (250) and $\Delta \boldsymbol{u}^{\prime}=\left[u_{x}^{\prime}, u_{y}^{\prime}, u_{z}^{\prime}\right]$ Equation (251). It is obvious that in this particular case there are huge differences between them.

Now when the distance between the sun and a star and relative velocity $\Delta \boldsymbol{u}$ of the star regarding the sun are known, we are able to derive values for proper motion [Equations (223) and (225)] and parallax [Equation (228)] and compare them with the values obtained by actual measurements. In this particular case, the differences between the derived and the actual values are enormous and because of that we didn't show them.

Our assumption is that these errors are mainly caused by using the data that do not fully suit the proposed method.

Table 6. Distance between the Earth and the star and star velocity calculated on the basis of the data obtained from the Gaia catalogs.

\begin{tabular}{|c|c|c|c|c|c|}
\hline Gaia source ID & 1996596911406176000 & 1267906854386665088 & 219999464832627584 & 932222445438498944 & 3471190026007380992 \\
\hline RA2015 [degrees] & 345.2287148122942 & 225.83211458394337 & 57.64076463233877 & 124.98562499895499 & 187.33090960579008 \\
\hline DEC2015 [degrees] & 54.38920810592237 & 25.42448994720175 & 36.11354636758435 & 50.884152757844795 & -30.842056520510575 \\
\hline RA2015.5 [degrees] & 345.2286881349424 & 225.83212885046885 & 57.640787372575275 & 124.98564294506525 & 187.33095326939153 \\
\hline DEC2015.5 [degrees] & 54.38919072361569 & 25.424567002148986 & 36.11352384195696 & 50.88412887441873 & -30.842103480719885 \\
\hline Parallax [mas] & 38.45223658078517 & 154.92079559457247 & -0.1736208834797856 & 3.825486423654411 & 24.626355359460785 \\
\hline $\mathrm{PM}(\mathrm{RA})$ [mas/year] & -110.82924669719277 & 87.72181780153205 & 148.0180947897536 & 81.4438996869589 & 269.2027204879181 \\
\hline $\mathrm{PM}(\mathrm{DEC})$ [mas/year] & -125.75137410621457 & 559.2198769511647 & -162.08048821185827 & -172.20093572981995 & -337.8374762654241 \\
\hline Radial velocity $[\mathrm{km} / \mathrm{sec}]$ & -22.001165161166835 & - & - & -0.2803438886939773 & 15.533125166544002 \\
\hline Distance(PRX) [km] & 8.0247 E14 & 1.991777 E14 & - & 8.0661 E15 & $1.252998 \mathrm{E} 15$ \\
\hline $\operatorname{Distance}(v=0)[\mathrm{km}]$ & $2.4297 \mathrm{E} 16$ & $1.8591 \mathrm{E} 16$ & 4.664 E15 & $3.5331 \mathrm{E} 17$ & $1.516 \mathrm{E} 17$ \\
\hline$u_{x}^{\prime} \quad[\mathrm{km} / \mathrm{sec}]$ & -7.028 & - & - & -489.140 & 2333.50 \\
\hline$u_{y}^{\prime} \quad[\mathrm{km} / \mathrm{sec}]$ & -15.606 & - & - & 48.835 & -3876.28 \\
\hline$u_{z}^{\prime} \quad[\mathrm{km} / \mathrm{sec}]$ & -18.786 & - & - & -425.58 & -1927.30 \\
\hline$u_{x} \quad[\mathrm{~km} / \mathrm{sec}]$ & $-165,355.38$ & - & - & $101,136.76$ & $132,912.72$ \\
\hline$u_{y} \quad[\mathrm{~km} / \mathrm{sec}]$ & $-54,985.63$ & - & - & $-219,887.01$ & $42,807.88$ \\
\hline$u_{z} \quad[\mathrm{~km} / \mathrm{sec}]$ & $-236,414.11$ & - & - & $-142,649.10$ & $63,517.15$ \\
\hline
\end{tabular}


Table 7. Distance between the Earth and the star and star velocity calculated on the basis of the data obtained from the Gaia catalogs.

\begin{tabular}{|c|c|c|c|c|c|}
\hline Gaia source ID & 3968823147582581504 & 3506524893749579648 & 2392703946771797248 & 1854353916756790400 & 3945681073517688960 \\
\hline RA2015 [degrees] & 164.37525525103945 & 200.09638529784812 & 350.6790229293687 & 321.88591137228934 & 185.63609385569734 \\
\hline DEC2015 [degrees] & 13.653681201911555 & -20.06343186628493 & -19.691300195639307 & 34.0238623055378 & 16.357596564626736 \\
\hline RA2015.5 [degrees] & 164.37528560226255 & 200.09640104426754 & 350.67898899365645 & 321.8858646334176 & 185.63607166653534 \\
\hline DEC2015.5 [degrees] & 13.653657150053572 & -20.063447680691795 & -19.6913255777869 & 34.02383684263199 & 16.3575826008146 \\
\hline Parallax [mas] & 20.911752813322053 & 11.496169556523695 & 23.25135907901302 & 38.45223658078517 & 15.40129228431169 \\
\hline $\mathrm{PM}(\mathrm{RA})[$ mas/year $]$ & 211.58298658446174 & 105.17501228068048 & -229.9247639971531 & -280.0377248573189 & -153.87591844804413 \\
\hline $\begin{array}{l}\mathrm{PM}(\mathrm{DEC}) \\
{[\text { mas/year] }}\end{array}$ & -172.99188421088598 & -113.83221442757147 & -183.05964366990693 & -182.44201795818088 & -100.88155554235291 \\
\hline $\begin{array}{l}\text { Radial velocity } \\
\qquad[\mathrm{km} / \mathrm{sec}]\end{array}$ & -39.2822790770779 & 4.635329233298119 & 33.98144938403769 & -67.36320930350523 & \\
\hline Distance(PRX) [km] & $1.47557 \mathrm{E} 15$ & $2.684 \mathrm{E} 15$ & $1.327 \mathrm{E} 15$ & 8.0247 E14 & 2.0035186 E15 \\
\hline Distance $(v=0)[\mathrm{km}]$ & $1.06517 \mathrm{E} 17$ & $5.857 \mathrm{E} 16$ & $1.3055 \mathrm{E} 17$ & $2.660 \mathrm{E} 16$ & 2.994104 E18 \\
\hline$u_{x}^{\prime} \quad[\mathrm{km} / \mathrm{sec}]$ & -185.815 & 22.296 & -38.842 & -58.269 & -20.272 \\
\hline$u_{y}^{\prime} \quad[\mathrm{km} / \mathrm{sec}]$ & -576.369 & -45.795 & -201.341 & -32.992 & 60.918 \\
\hline$u_{z}^{\prime} \quad[\mathrm{km} / \mathrm{sec}]$ & -183.643 & -24.109 & -60.015 & -52.323 & -93.284 \\
\hline$u_{x}[\mathrm{~km} / \mathrm{sec}]$ & $240,711.77$ & $253,830.47$ & $-268,636.98$ & $-188,091.10$ & $285,858.14$ \\
\hline$u_{y} \quad[\mathrm{~km} / \mathrm{sec}]$ & $-86,558.65$ & $124,417.50$ & $78,995.59$ & $71,127.52$ & -7594.04 \\
\hline$u_{z} \quad[\mathrm{~km} / \mathrm{sec}]$ & $-29,133.97$ & $53,599.13$ & $71,777.99$ & $-206,760.47$ & $-88,673.82$ \\
\hline
\end{tabular}

In order to improve the results we should know the exact coordinates of the the points $A, B$ and $C$ actually the locations of the satellite at the moments when the measurements have been made. The coordinate systems and transformation matrices should be replaced by the appropriate ones. The reference system for the source catalog is the Barycentric Celestial Reference System (BCRS/ICRS), but for the proposed method it is necessary that the Gaia observations are expressed in the Centre-of-Mass Reference System (CoMRS), a system that moves with the Gaia spacecraft.

Therefore, for all these reasons, the results shown in (Table 6) and (Table 7) must be taken with great caution.

Please refer to the attached program "gaia0.wxmx" for further explanations and data testing. In this program is also given a code for extracting data from Gaia's databases.

\section{Determining a Velocity $v$ regarding the $(\mathrm{K})$}

It has already been mentioned why, in the case of very distant objects, it is important that the velocity $v$ is known. One of the methods for determining the velocity $v$ is given in the [7], where the problem of the possible relation between absolute velocity and stellar aberration has been discussed. 
In the rest of this section, we present another a method which possibly may serve in determining the velocity $\boldsymbol{v}$. Equations (166)-(168) can be written in modified forms, where $\Delta u_{x}, \Delta u_{y}$ and $\Delta u_{z}$ are derived from the Equation (249).

$$
\begin{gathered}
\Delta u_{x}+\epsilon_{x}=\frac{C_{x}-A_{x}+c_{x} * d_{3}-a_{x} * d_{1}}{\Delta T_{2}}-v_{x} \\
\Delta u_{y}+\epsilon_{y}=\frac{C_{y}-A_{y}+c_{y} * d_{3}-a_{y} * d_{1}}{\Delta T_{2}}-v_{y} \\
\epsilon_{x}=c * \frac{u_{z}+\epsilon_{z}=\frac{C_{z}-A_{z}+c_{z} * d_{3}-a_{z} * d_{1}}{\Delta T_{2}}-v_{z}}{\Delta t_{2} * v_{x}+R *\left(\cos \left(\frac{2 \Pi * t_{3}}{y e a r s e c}\right)-\cos \left(\frac{2 \Pi * t_{1}}{y e a r s e c}\right)\right)+c_{x} * d_{3}\left(v_{x}, v_{y}, v_{z}\right)-a_{x} * d_{1}\left(v_{x}, v_{y}, v_{z}\right)} \\
\epsilon_{y}=c * \frac{d_{1}\left(v_{x}, v_{y}, v_{z}\right)-d_{3}\left(v_{x}, v_{y}, v_{z}\right)+c * \Delta t_{2}}{\Delta t_{2} * v_{y}+R *\left(\sin \left(\frac{2 \Pi * t_{3}}{y e a r s e c}\right)-\sin \left(\frac{2 \Pi * t_{1}}{y e a r s e c}\right)\right)+c_{y} * d_{3}\left(v_{x}, v_{y}, v_{z}\right)-a_{y} * d_{1}\left(v_{x}, v_{y}, v_{z}\right)} \\
d_{1}\left(v_{x}, v_{y}, v_{z}\right)-d_{3}\left(v_{x}, v_{y}, v_{z}\right)+c * \Delta t_{2} \\
\epsilon_{z}=c * \frac{\Delta t_{2} * v_{z}+c_{z} * d_{3}\left(v_{x}, v_{y}, v_{z}\right)-a_{z} * d_{1}\left(v_{x}, v_{y}, v_{z}\right)}{d_{1}\left(v_{x}, v_{y}, v_{z}\right)-d_{3}\left(v_{x}, v_{y}, v_{z}\right)+c * \Delta t_{2}}-\Delta u_{z}-v_{z}
\end{gathered}
$$

We can form a function $g\left(v_{x}, v_{y}, v_{z}\right)$ in the following way

$$
g\left(v_{x}, v_{y}, v_{z}\right)=\epsilon_{x}^{2}+\epsilon_{y}^{2}+\epsilon_{z}^{2}
$$

Now we can try to find a minimum value of the the function $g\left(v_{x}, v_{y}, v_{z}\right)$ with respect to unknowns $v_{x}, v_{y}$ and $v_{z}$. If there are such $v_{x 0}, v_{y 0}$ and $v_{z 0}$ for which $g\left(v_{x}, v_{y}, v_{z}\right)$ has a minimum, then we can say that Equation (266) has a solution.

Let us suppose that we have observed $n$ stars from our Galaxy. Corresponding velocities, obtained by Equation (266) are marked by $\boldsymbol{v}_{1}, \boldsymbol{v}_{2}, \cdots, \boldsymbol{v}_{n}$.

Let define $\boldsymbol{v}_{0}$ as a mean value of the the vectors $\left\{\boldsymbol{v}_{i}\right\}$

$$
\boldsymbol{v}_{0}=\frac{\sum_{i=1}^{n} \boldsymbol{v}_{i}}{n}
$$

Let $\varepsilon$ is some small positive number.

If the following inequality hold

$$
\frac{\sum_{i=1}^{n}\left|\boldsymbol{v}_{i}-\boldsymbol{v}_{0}\right|}{n}<\varepsilon
$$

then we will consider two cases

1) $\boldsymbol{v}_{0}=\mathbf{0}$

it is only possible to determine a velocity of a star relative to the sun

2) $\left|v_{0}\right|>0$

Let $\boldsymbol{v}_{k}$ is defined by Equation (38) and 
an $\varepsilon_{1}$ is some small positive number.

We will consider two subcases:

a) if $\left|\boldsymbol{v}_{0}-\boldsymbol{v}_{k}\right| \leq \varepsilon_{1}$ then $\boldsymbol{v}_{0}$ represents velocity by which the Sun moves relative to the Galactic barycenter

b) if $\left|\boldsymbol{v}_{0}-\boldsymbol{v}_{k}\right|>\varepsilon_{1}$ then $\boldsymbol{v}_{0}$ represents velocity by which the Sun moves regarding the stationary frame $(K)$

If an inequality (268) does not hold then this approach to finding the velocity of the solar system regarding the Galactic barycenter (or a velocity $\mathbf{v}$ regarding the stationary frame $(K)$ ) did not yield the expected results and therefore it must be rejected.

The same procedure can be repeated, but this time the extra-galactic objects should be observed.

\section{Conclusion}

In this paper, we have developed and tested a method by which it is possible to find the distance $d$ between the sun and the arbitrary cosmic object as well as the velocity $\Delta \boldsymbol{u}$ at which this object moves relative to the sun. The advantage of this method in comparison to the traditional parallax method is that it can be applied to very distant objects, provided that the measurements are performed over a long period of time and that the velocity at which the solar system moves relative to the coordinate system $(\mathrm{K})$ is known. In addition to distance $d$ and velocity $\Delta \boldsymbol{u}$, it is also possible to derive values for proper motion, parallax and radial velocity and compare them with values obtained by direct measurements. In this way, we are able to assess to what extent the results obtained by this method are correct.

\section{Conflicts of Interest}

The author declares no conflicts of interest regarding the publication of this paper.

\section{References}

[1] Lindegren, L., et al. (2016) Gaia Data Release 1. https://www.aanda.org/articles/aa/pdf/2016/11/aa28714-16.pdf

[2] The Hipparcos and Tycho Catalogues (1997) Astrometric and Photometric Star Catalogues.

https://www.cosmos.esa.int/documents/532822/552851/vol1_all.pdf/99adf6e3-68934824-8fc2-8d3c9cbba2b5

[3] Majewski, S. (2016) Coordinate Systems. http://www.faculty.virginia.edu/ASTR5610/lectures/COORDS/coords.html

[4] Maxima, a Computer Algebra System. Version 17.10.1 (2017). http://maxima.sourceforge.net

[5] Fabricius, C., et al. (2016) Gaia Data Release 1. https://www.aanda.org/articles/aa/pdf/2016/11/aa28643-16.pdf

[6] (2018) Gaia Archive. https://gea.esac.esa.int/archive/ 
[7] Čojanović, M. (2018) Absolute Velocity and Total Stellar Aberration. Journal of Applied Mathematics and Physics, 6, 1034-1054.

https://doi.org/10.4236/jamp.2018.65090 